Supporting Information

\title{
Ultrafast Synthesizing Bismuth Mesoporous Nanolitchi Radiosensitizer Loading High Dose DOX for CT Guided Enhanced \\ Chemoradiotherapy
}

Jie Liu ${ }^{a}$, Yong Deng ${ }^{a}$, Xiaojia Qin $^{b}$, Bing Li ${ }^{b}$, Jianping Zhang ${ }^{b, d}$, Yunhua Xu ${ }^{c}$, Ruizhuo Ouyang $^{a}$,Yuhao Li ${ }^{a *}$, Yuqing Miao ${ }^{a *}$, Yun Sun ${ }^{a, b, d, e *}$

a Institute of Bismuth Science \& College of Science, University of Shanghai for Science and Technology, Shanghai 200093, China.

b Department of Research and Development \& Department of Nuclear Medicine, Shanghai Proton and Heavy Ion Center, Fudan University Shanghai Cancer Center, Shanghai 201321, China.

c Shanghai Lung Cancer Center, Shanghai Chest Hospital, Shanghai Jiao Tong University, Shanghai 200030, China.

d Shanghai Engineering Research Center of Proton and Heavy Ion Radiation Therapy, Shanghai 201321, China.

e Shanghai Engineering Research Center for Molecular Imaging Probes, Shanghai 200032, China.

Corresponding Authors:

yhli@usst.edu.cn (Y. Li)

yqmiao@usst.edu.cn (Y. Miao)

yun.sun@sphic.org.cn (Y.Sun) 


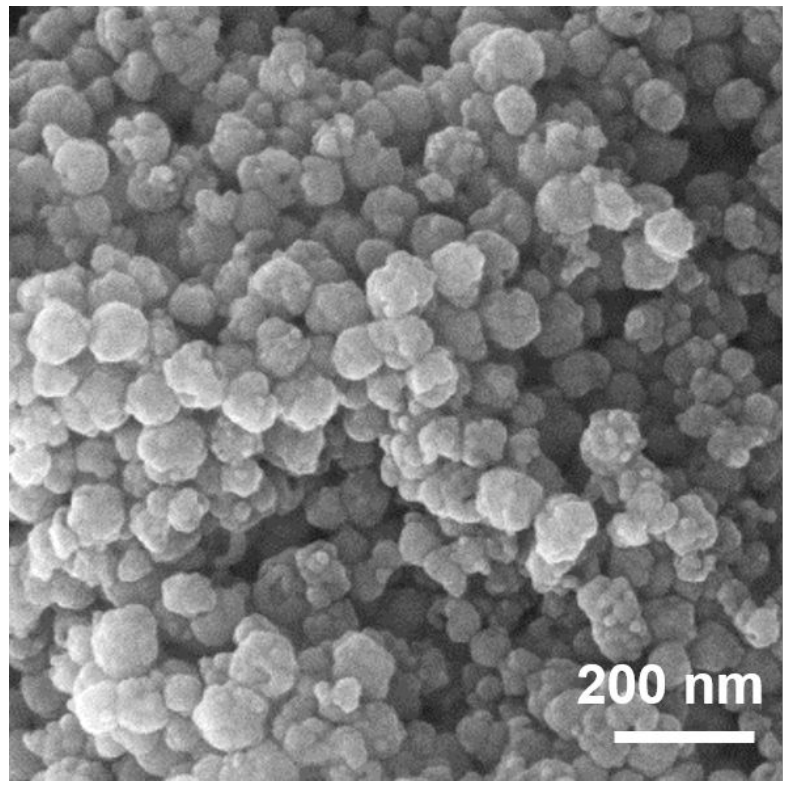

Figure S1. SEM image of NBOF NPs.

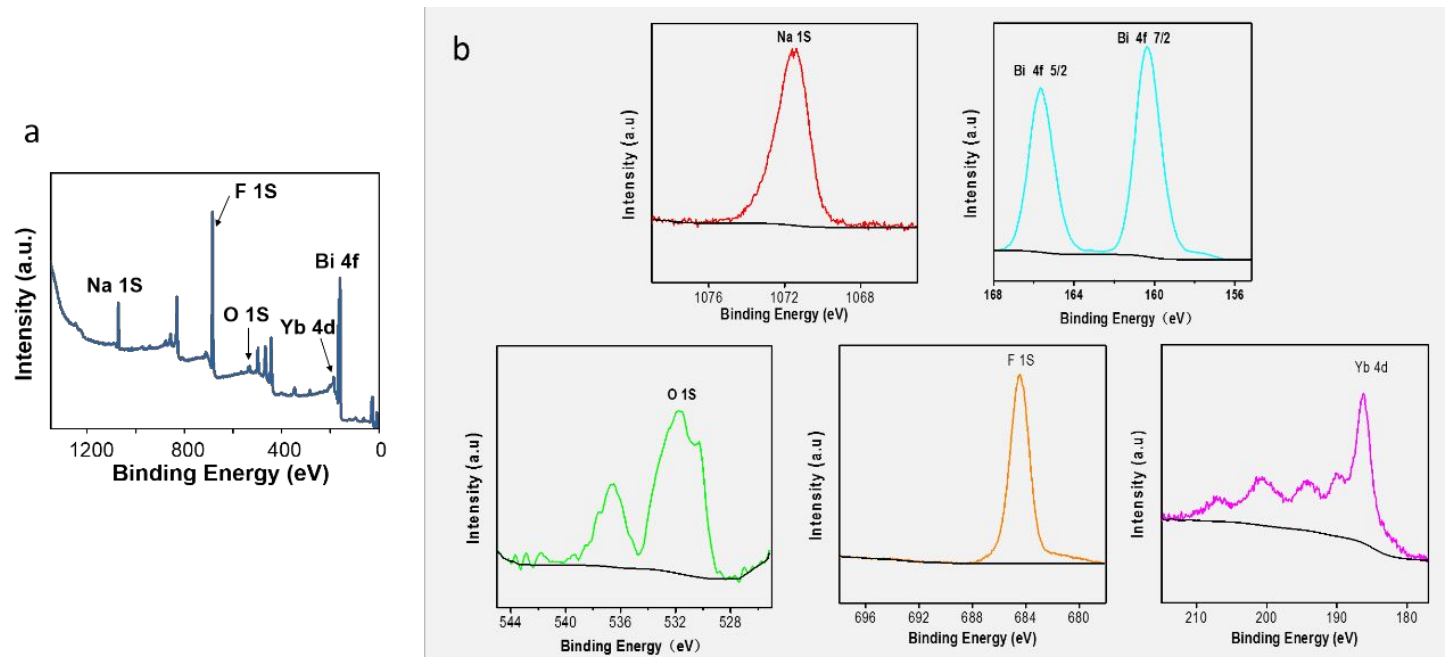

Figure S2. Characterization of NBOF (a) XPS data, (b) enlarged XPS patterns of different elements. 


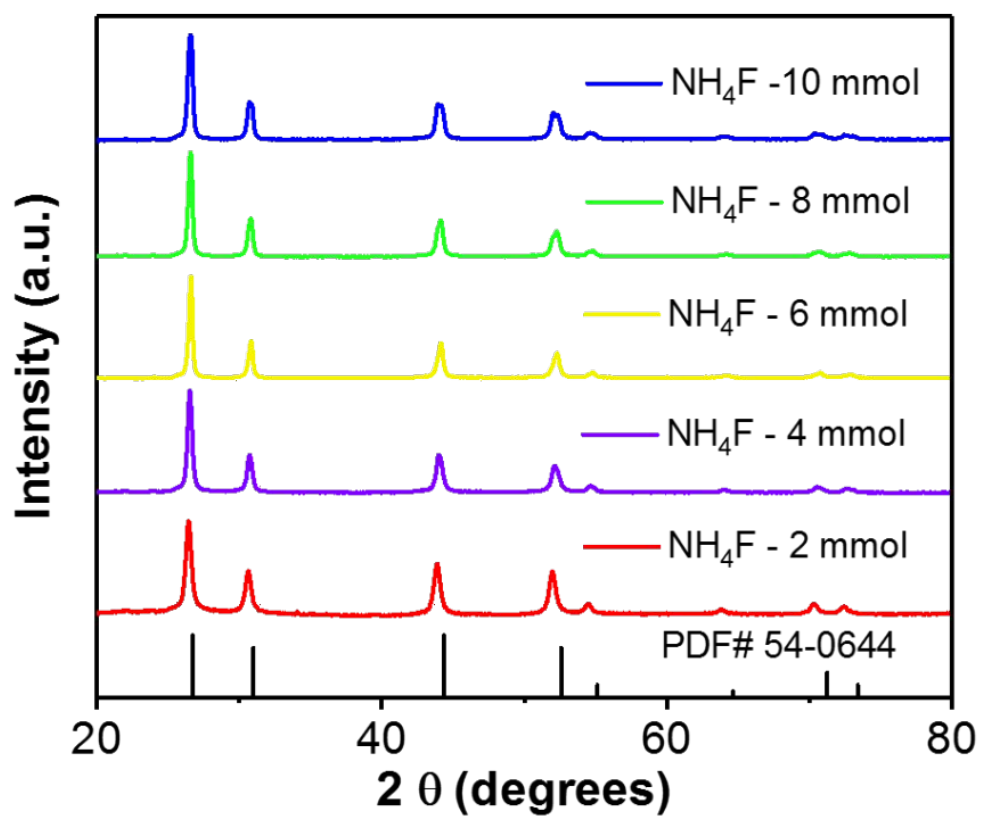

Figure S3. XRD patterns in different $\mathrm{NH}_{4} \mathrm{~F}$ concentrations (general reaction condition and post-treatment with $\mathrm{H}_{2} \mathrm{O}$ ).

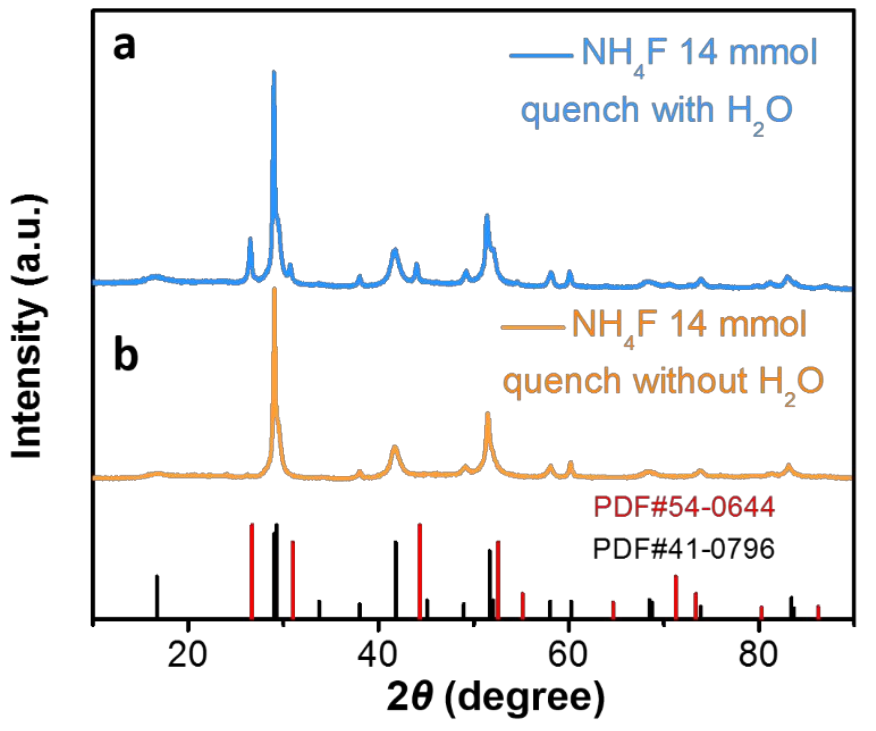

Figure S4. XRD patterns in $14 \mathrm{mmol} \mathrm{NH}_{4} \mathrm{~F}$ concentration (general reaction condition and quenched with (a) or without (b) $\mathrm{H}_{2} \mathrm{O}$ ). 


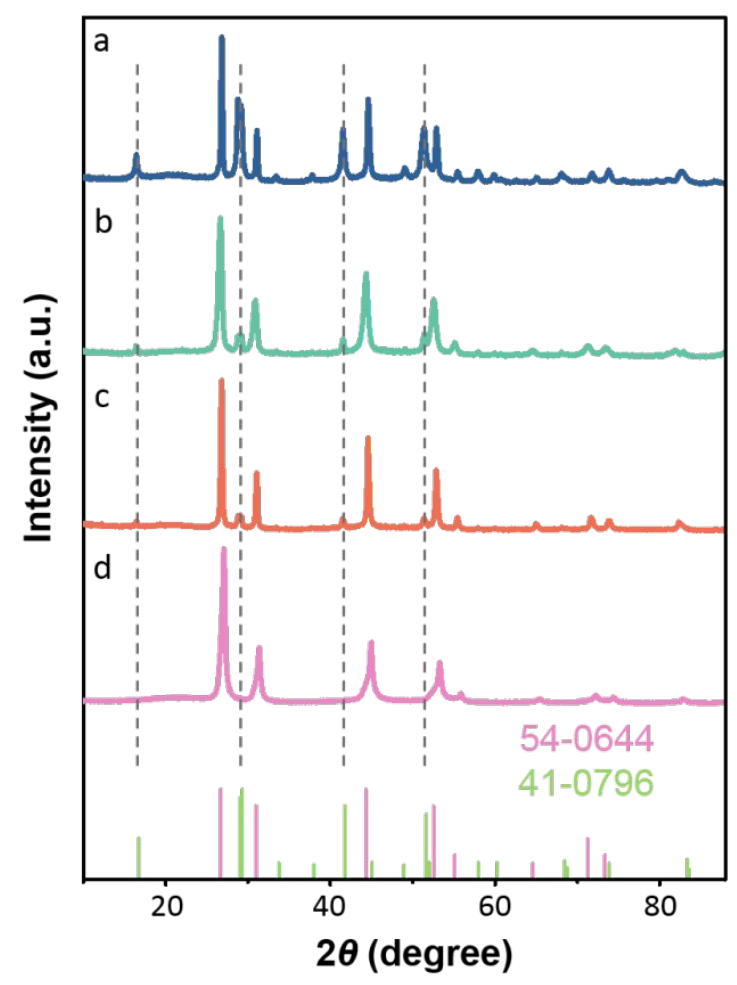

Figure S5. XRD patterns in different reaction conditions (a) anaerobic reaction condition, (b) anhydrous and anaerobic reaction condition, (c) general reaction condition. For (a), (b) and (c) reaction, the post-treatment without $\mathrm{H}_{2} \mathrm{O}$ and (d) general reaction condition and post-treatment with $\mathrm{H}_{2} \mathrm{O}$.

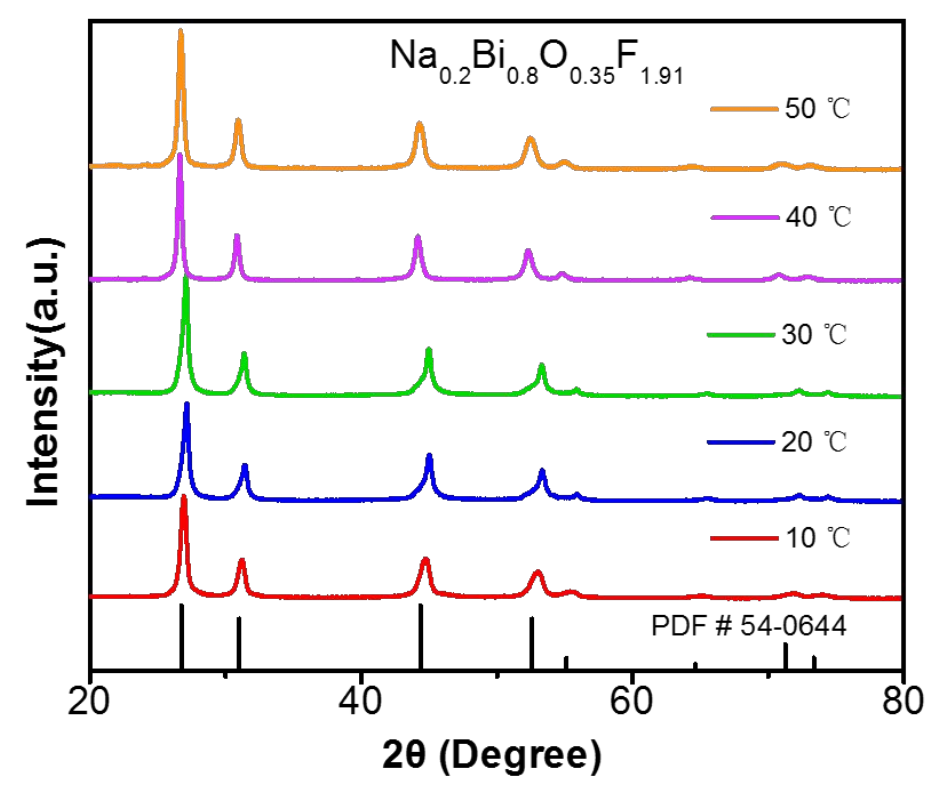

Figure S6. XRD patterns in different reaction temperature (general reaction condition and post-treatment with $\mathrm{H}_{2} \mathrm{O}$ ). 


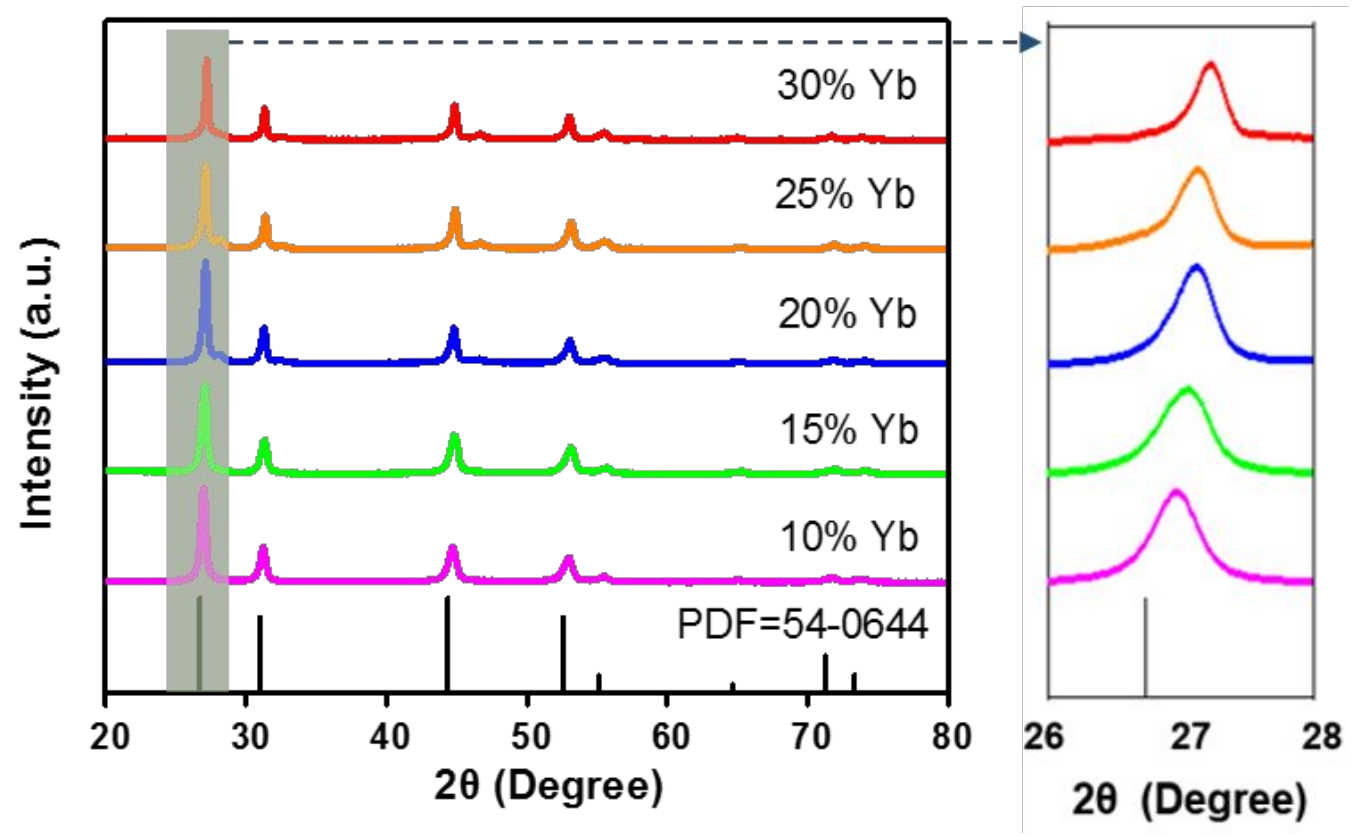

Figure S7. XRD patterns in different $\mathrm{Yb}$ doped concentration (general reaction condition and post-treatment with $\mathrm{H}_{2} \mathrm{O}$ ).

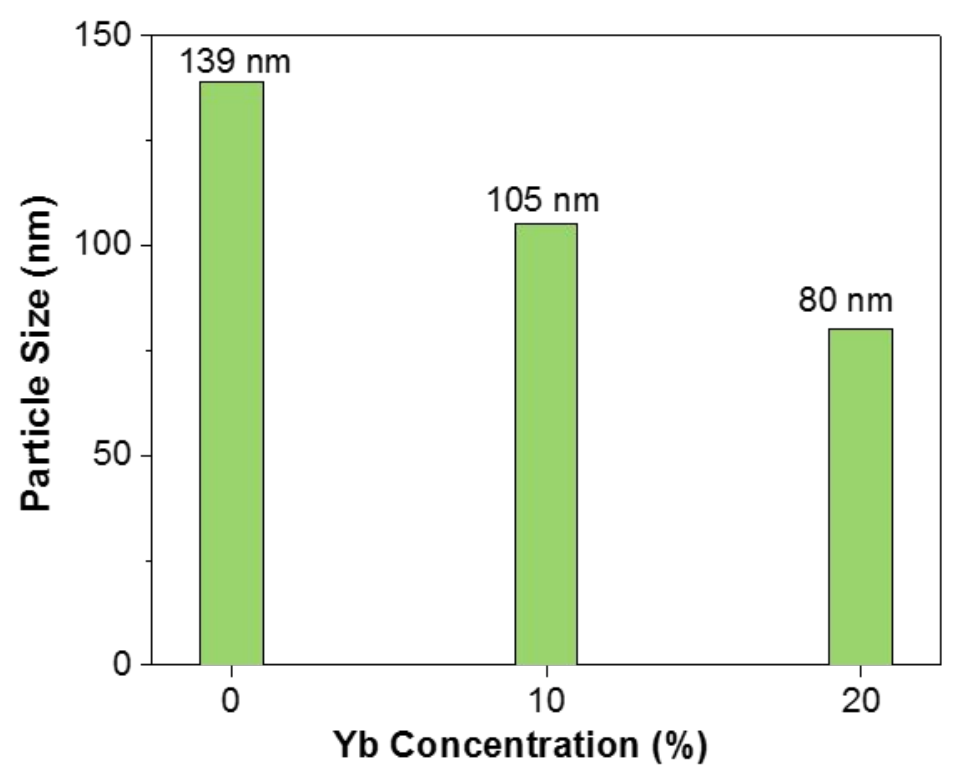

Figure S8. The comparison of the average particle size obtained under different $\mathrm{Yb}$ ion doped concentration. 


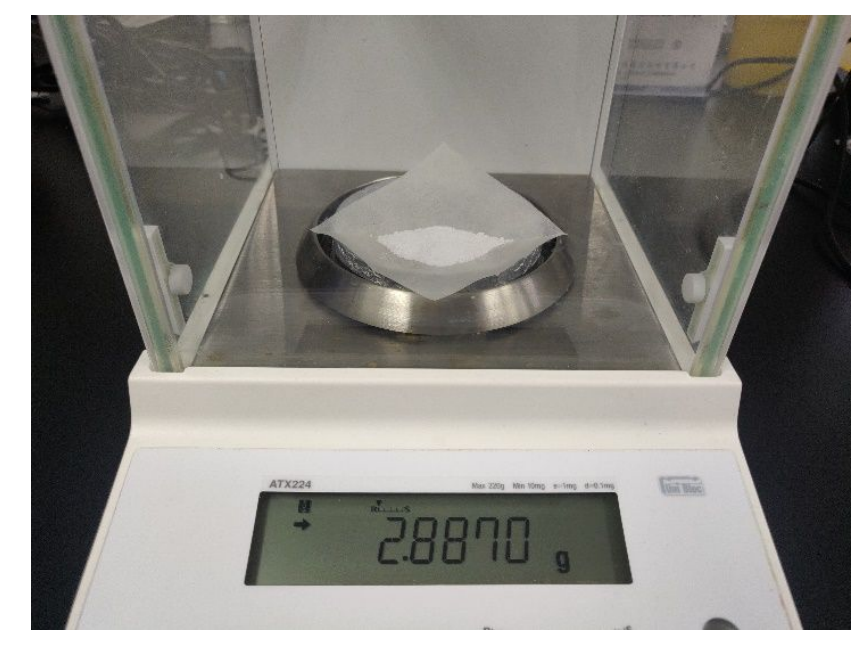

Figure S9. Digital photo of the quality of NBOF NPs.

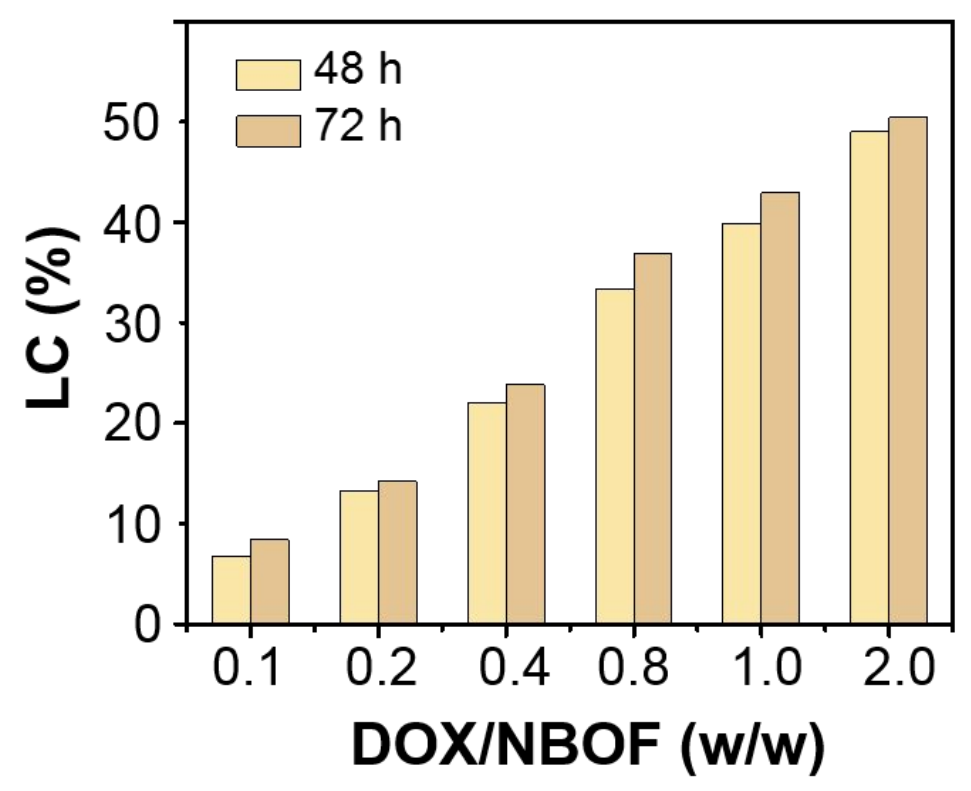

Figure S10. DOX loading capacity (LC) in different raw materials ratio between DOX and NBOF. 


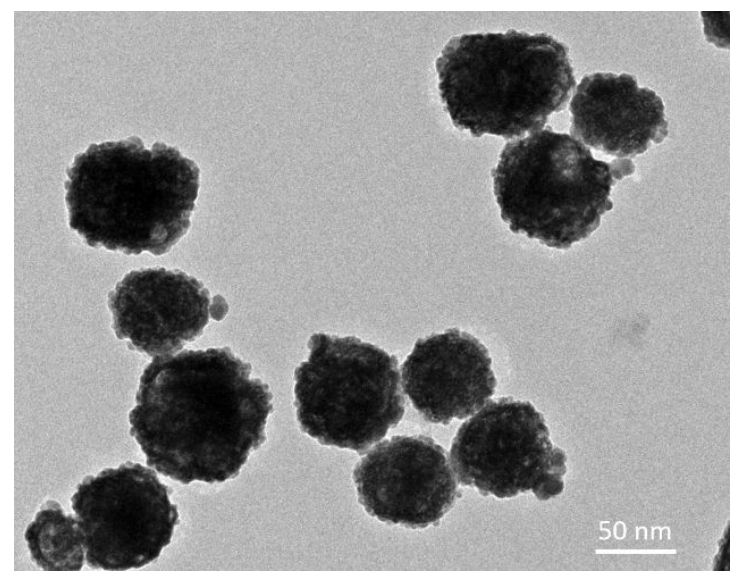

Figure S11. TEM image of NBOF-DOX.
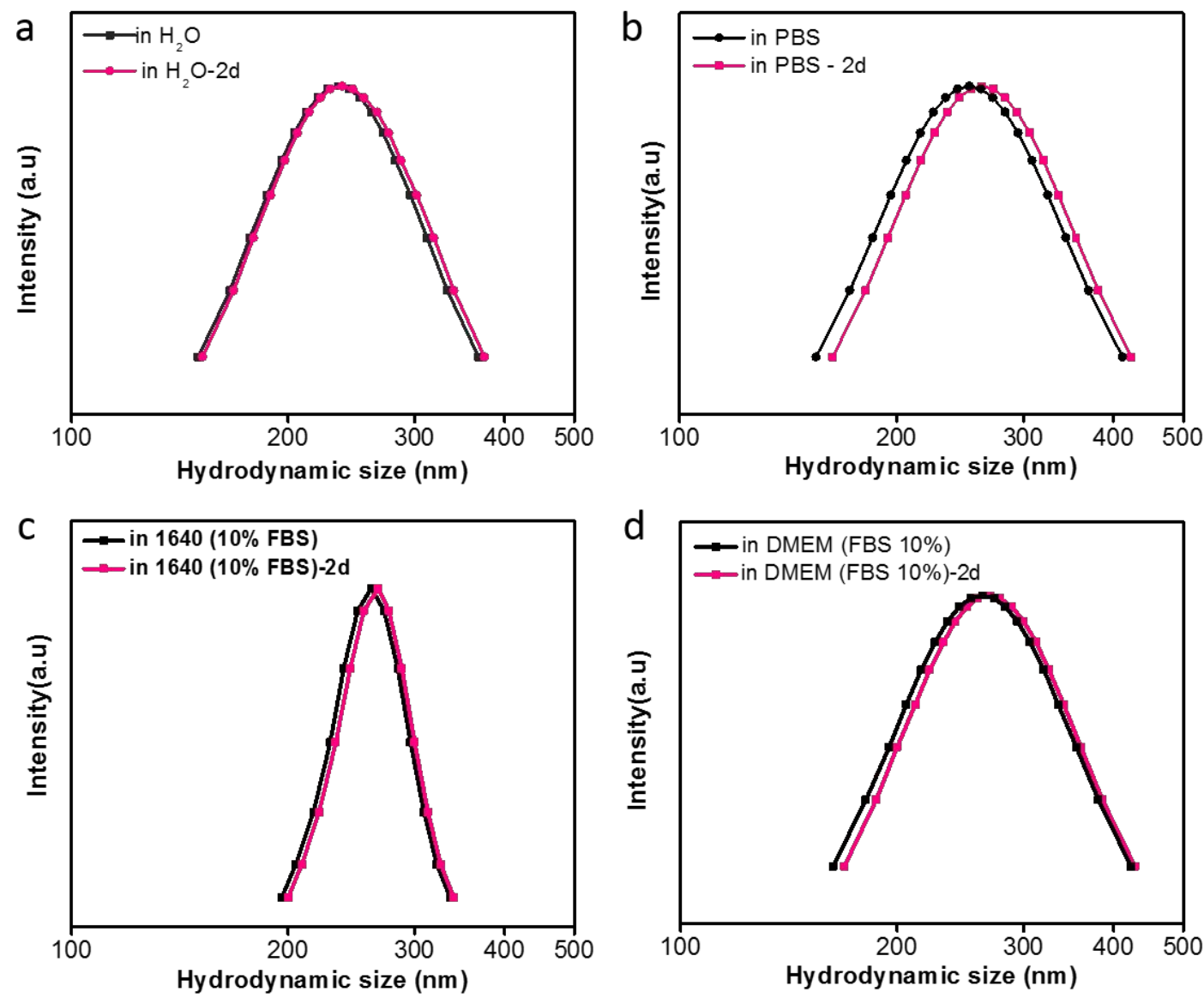

Figure S12. DLS of NBOF-DOX-PEG NPs in different solutions for $0 \mathrm{~h}$ and 2 days (2 d) (a) $\mathrm{H}_{2} \mathrm{O}$, (b) PBS, (c) 1640 culture medium with 10\% FBS, and (d) DMEM with $10 \%$ FBS. 


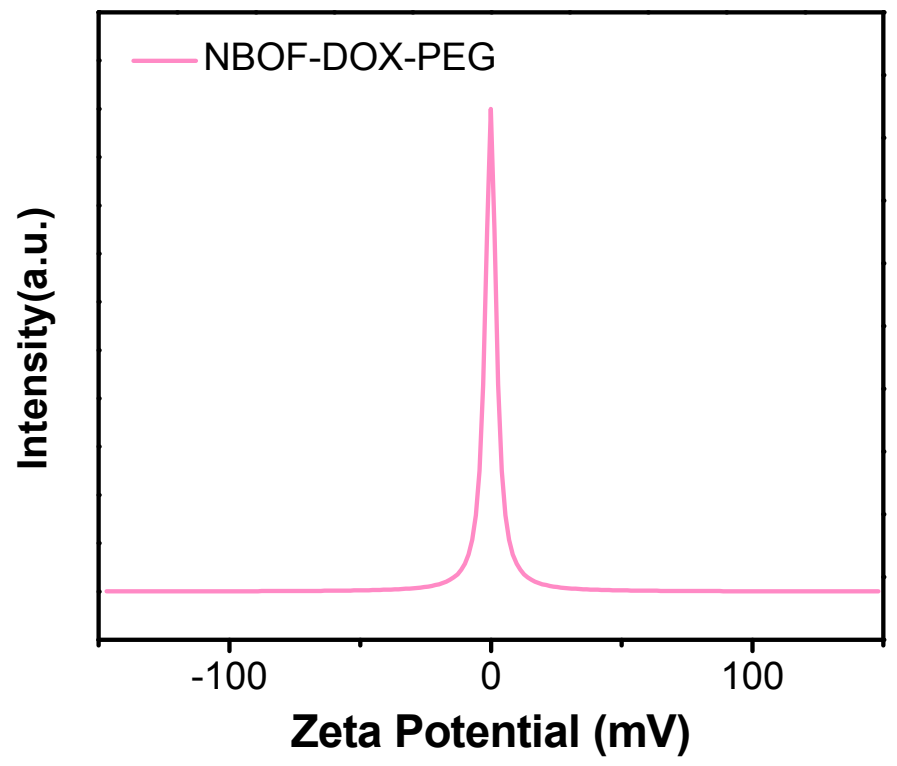

Figure S13. Zeta potential of NBOF-DOX-PEG NPs in $\mathrm{H}_{2} \mathrm{O}$.

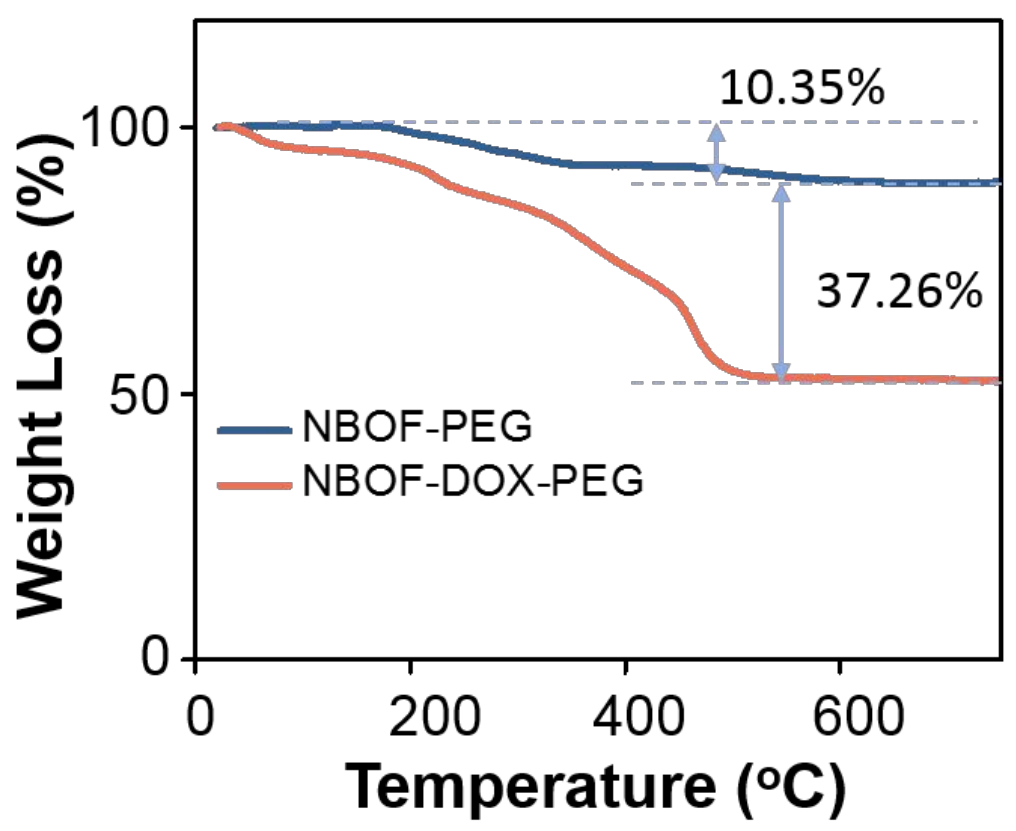

Figure S14. TG data of NBOF-PEG and NBOF-DOX-PEG. 


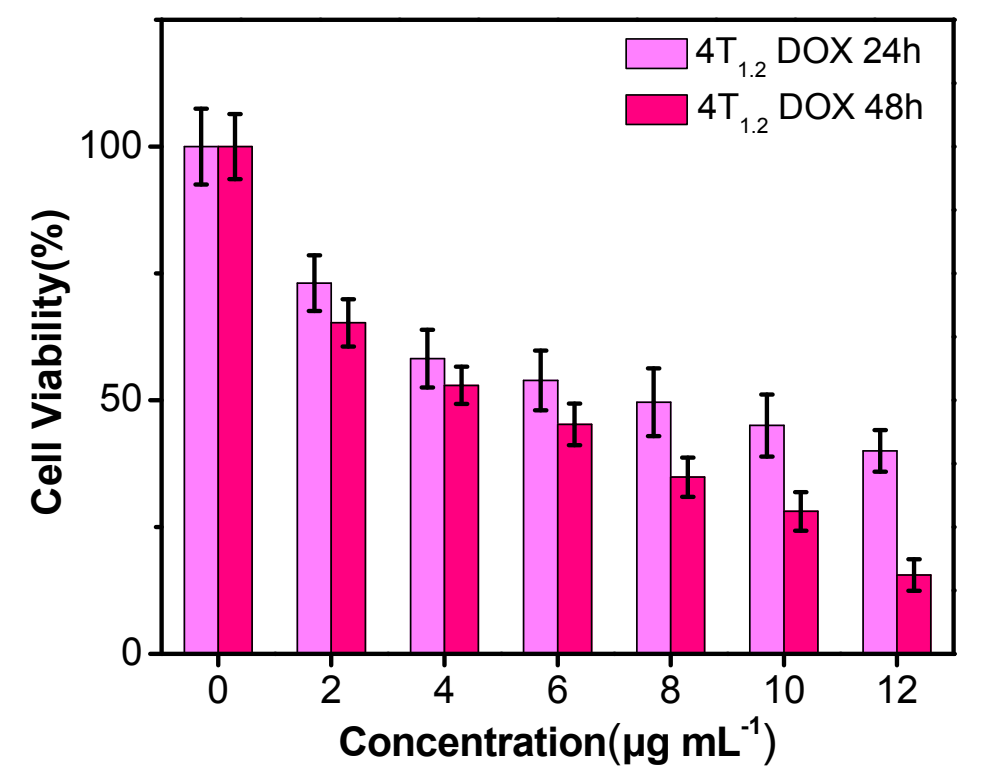

Figure S15. CCK8 results of $4 \mathrm{~T}_{1.2}$ cells incubated with DOX in different concentration for 24 and $48 \mathrm{~h}$.

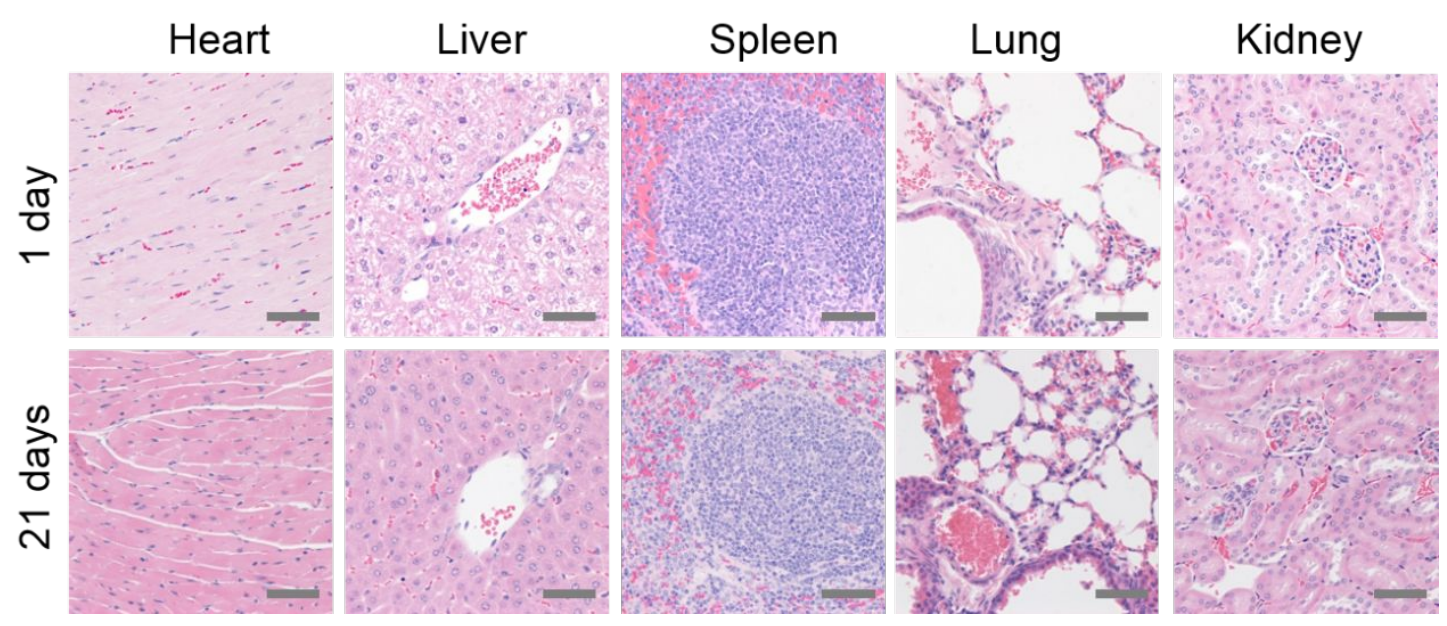

Figure S16. H\&E stained images of different mice organs treated with NBOF-PEG for 1 day and 21 days (scale bar $100 \mu \mathrm{m}$ ). 

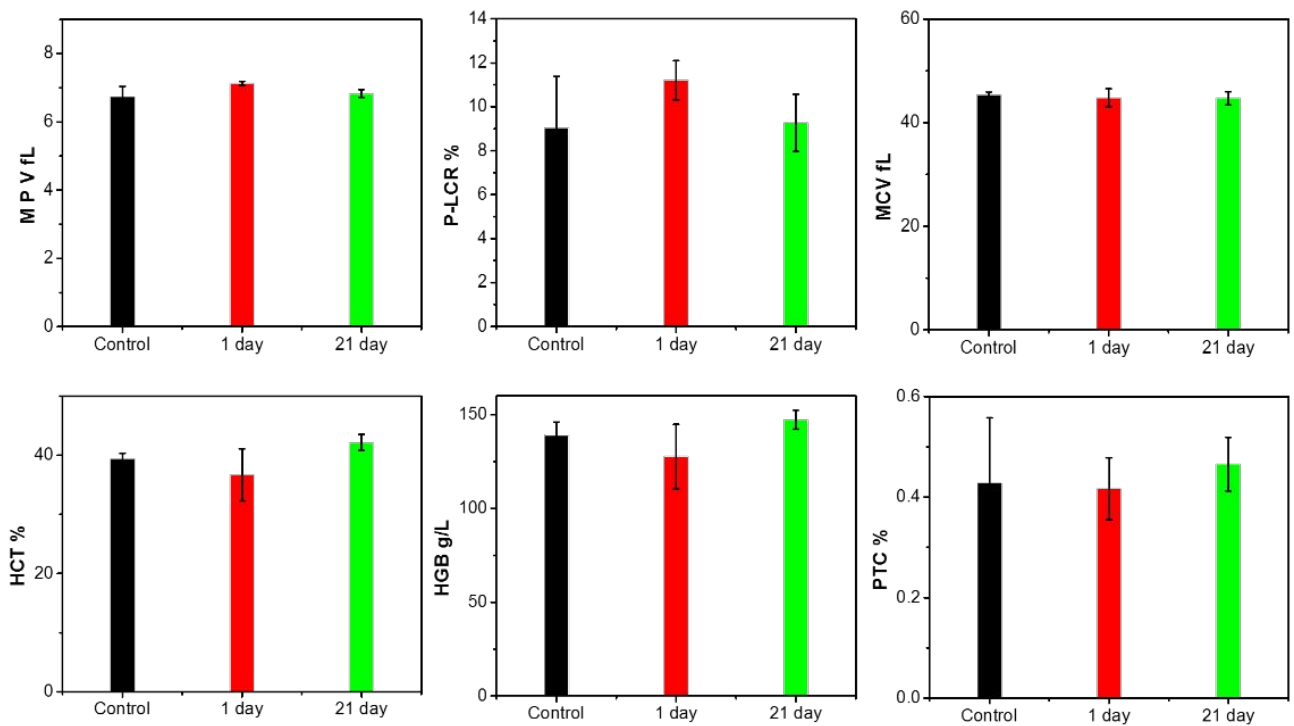

Figure S17. Blood analysis data of healthy BALB/c mice were intravenous (i.v.) injected NBOF-PEG $\left(4 \mathrm{mg} \mathrm{kg}^{-1}, 200 \mu \mathrm{L}\right)$ after 1 day or 21 days. Healthy BALB/c mice were intravenous (i.v.) injected saline $(200 \mu \mathrm{L})$ as control. 